BULL. AUSTRAL. MATH. SOC.

VOL. 9 (1973), 61-68.

\title{
On categorical semigroups
}

\section{R.A.R. Monzo}

A semigroup $S$ is called categorical if every ideal $I$ of $S$ has the property that $a b c \in I \quad(a, b, c \in S)$ implies $a b \in I$ or $b c \in I$. Necessary and sufficient conditions for an orthodox semigroup to be categorical are found and then used to characterize those bands which appear as an isomorphic copy of the band of idempotents of some orthodox categorical semigroup and to simplify the proof of a theorem of Mario Petrich. The structure of commutative categorical semigroups is found modulo the structure of abelian groups and categorical semilattices.

\section{Orthodox categorical semigroups}

Categorical semigroups have been studied by Petrich [1] and in the commutative case by McMorris and Satyanarayana [5] and the author [6]. We will assume the reader is familier with Green's relations and with the terminology used in [5].

The following two results were proven in [6]:

PROPOSITION 1. A semilattice is categorical if and only if it is a tree.

PROPOSITION 2. A regular semigroup $S$ is categorical if and only if its set of idempotents $E_{S}$ satisfies the following property:

if $e, f, g \in E_{S}, e \leq g$ and $f \leq g$, then $e \in J(e f)$ or

$f \in J(e f)$.

An orthodox semigroup is a regular semigroup in which the set of idempotents is a subsemigroup.

Received 6 March 1973. Communicated by S.A. Huq. 
PROPOSITION 3. An orthodox semigroup $s$ is categorical if and only if whenever $e \leq g$ and $f \leq g$ for some $e, f, g \in E_{S}$, there exists $t \in E_{S}$ such that $t \leq e f$ and either tDe or tof.

Proof. Suppose that $S$ is an orthodox categorical semigroup. Let $e \leq g$ and $f \leq g$ for some $e, f, g \in E_{S}$. By Proposition 2, either $e \in J(e f)$ or $f \in J(e f)$. Assume $e \in J(e f)$. Then $e=a e f b$ for some $a, b \in S^{\prime}$. Let $t=$ efbeaef. Then $t$ is idempotent, $t \leq e f$, and tDe. Similarly, if $f \in J(e f)$ then there exists an idempotent $t \leq e f$ such that $t D f^{\circ}$. This proves the necessity of the proposition. Since $D \subseteq J$ the sufficiency follows from Proposition 2.

PROPOSITION 4. If $e, f \in E_{S}$ and $e D_{f}$ then $e E_{S} e \cong f E_{S} f$.

REMARK. This last result is proven by Baird [1] for an arbitrary semigroup $S$. If $E_{S}$ is not a band then $\cong$ is to be interpreted as meaning 'partially isomorphic to'. Since there exists a non-categorical semilattice $T$ with the property that $e E_{T} e \cong f E_{T} f$ for any $e, f \in E_{T}$ (cf. [6]) one sees that $e E_{S} e \cong f E_{S} f$ does not imply $e D f$ in general.

THEOREM 1. A band $B$ appecars as an isomorphic copy of $E_{S}$ for some or thodox categorical semigroup $S$ if and only if whenever $e \leq g$ and $f \leq g$ for some $e, f, g \in B$ then there exists $t \in B$ such that $t \leq$ ef and either $e B e \cong t B t$ or $f B f \cong t B t$.

Proof. The necessity of the theorem follows from Propositions 3 and 4. To prove sufficiency, assume that $B$ satisfies the property stated in the last part of the theorem. Hall has constructed an orthodox semigroup $W$ such that $B \cong E_{W}$ and such that $e E_{W} e \cong f E_{W} f$ (for some $e, f \in E_{W}$ ) if and only if $e D_{f}$ (cf. [4]). Identify $B$ with $E_{W}$. Suppose that $e \leq g$ and $f \leq g$ for some $e, f, g \in B$. Then there exists $t \in B$ such that $t \leq e f$ and either $t B t \cong e B e$ or $t B t \cong f B f$. Assume that $t B t \cong e B e$. Then $t D e$ and so $t J e$. Since $t \leq e f$ it follows that $e \in J(e f)$. Similarly, if $t B t \cong f B f$ then $f \in J(e f)$. By Proposition 2, $W$ is categorical. This completes the proof of the theorem. 


\title{
2. A new proof of Petrich's Theorem
}

\author{
A semigroup $S$ is called left-elementary if $L=D \cap \lambda$, where \\ $\lambda=\left\{(x, y) \in S \times S: S^{\prime} x \subseteq S^{\prime} y\right\}$.
}

THEOREM 2. A left-elementary inverse semigroup is categorical if and only if its semizattice of idempotents is categorical.

Proof. Suppose that $S$ is a left-elementary inverse semigroup which is also categorical. By Proposition 1, we need only show that $E_{S}$ is a tree. Let $e \leq g$ and $f \leq g$ for some $e, f, g \in E_{S}$. Then, by Proposition 3, there exists $t \in E_{S}$ such that $t \leq e f$ and either eDt or fDt. If eDt then $S^{\prime} t=S^{\prime} t e f=S^{\prime} t f e$ and so $S^{\prime} t \subseteq S^{\prime} e$. Since $S$ is left-elementary, $S^{\prime} t=S^{\prime} e$ and therefore $e \in S^{\prime} t e f$. This means that $e=e f$. Similarly, if $f D t$ then $f=e f$. We have thus shown that $E_{S}$ is a tree. This proves necessity, and sufficiency follows from Propositions 1 and 2 .

COROLLARY (Petrich [7]). A completely semisimple inverse semigroup is categorical if and only if it has a tree of idempotents.

REMARK. Hall [4] has proved that a regular left-elementary semigroup is completely semisimple.

\section{The structure of commutative categorical semigroups}

Let $S$ be a semigroup and for each $x \in S$ assign a set $A_{x}$ such that $x \in A_{x}$ and $A_{x} \cap A_{y}=\emptyset$ if $x \neq y$. Then $\underset{x \in S}{\cup} A_{x}$ becomes a semigroup if we define multiplication as:

$$
a_{x y} b_{y}=x y \text { for } a_{x} \in A_{x}, b_{y} \in A_{y} \text {. }
$$

We write $\left(\left(S, A_{x}\right)\right)$ for the semigroup constructed in the paragraph above. It is called an inflation of $S$.

A semigroup is called separative if $x^{2}=x y=y^{2}$ implies $x=y$.

THEOREM 3. Let $S=S^{2}, T=T^{2}$ and suppose $S$ and $T$ are separative. Then $\left(\left(S, A_{x}\right)\right)$ and $\left(\left(T, B_{y}\right)\right)$ are isomorphic if and only if 
there is an isomorphism $\phi: S+T$ such that $\left|A_{x}\right|=\left|B_{\phi(x)}\right|$ for all $x \in S$.

Proof. Let $\psi:\left(\left(S, A_{x}\right)\right)+\left(\left(T, B_{y}\right)\right)$ be an isomorphism and let $\phi$ be the restriction of $\psi$ to $S$. Since $S=S^{2}$ and $T=T^{2}, \phi(S)=T$ and $\phi$ is an isomorphism onto $T$.

Suppose $a \in A_{x}$ and $\psi(a) \in B_{t}$ for some $x \in S, t \in T$. Then $t^{2}=[\psi(a)]^{2}=\psi\left(a^{2}\right)=\psi\left(x^{2}\right)=[\psi(x)]^{2}$ and $\psi\left(x^{2}\right)=\psi(a x)=\psi(a) \psi(x)=t \psi(x)$. Since $T$ is separative, $t=\psi(x)$. We have shown that $\psi\left(A_{x}\right) \subseteq B_{\psi(x)}$. By symmetry, $\psi^{-1}\left(B_{\psi(x)}\right) \subseteq A_{x}$ and so $\psi\left(A_{x}\right)=B_{\psi(x)}=B_{\phi(x)}$ and $\left|A_{x}\right|=\left|B_{\phi(x)}\right|$.

This proves the necessity of the theorem.

Now suppose $\phi: S \rightarrow T$ is an isomorphism such that for all $x \in S$, $\left|A_{x}\right|=\left|B_{\phi(x)}\right|$. For all $x \in S$, let $\psi_{x}: A_{x} \rightarrow B_{\phi(x)}$ be a bijection such that $\psi_{x}(x)=\phi(x)$. Define $\psi:\left(\left(S, A_{x}\right)\right) \rightarrow\left(\left(T, B_{y}\right)\right)$ as $\psi(a)=\psi_{x}(a)$ for any $a \in A_{x}$. Then $\psi$ is an isomorphism.

This proves the sufficiency of the theorem and the proof of Theorem 3 is therefore complete.

Let $Y$ be a semilattice. For each $\alpha \in Y$ assign a group $G_{\alpha}$ such that $G_{\alpha} \cap G_{\beta}=\emptyset$ if $\alpha \neq \beta$. If $\alpha>\beta$ choose a homomorphism $\phi_{\alpha, \beta}: G_{\alpha} \rightarrow G_{\beta}$ such that if $\gamma<\beta<\alpha$ then $\phi_{\beta, \gamma} \phi_{\alpha, \beta}=\phi_{\alpha, \gamma}$. For $\alpha \in Y$ let $\phi_{\alpha, \alpha}$ be the identity on $G_{\alpha}$. Let $S_{Y}=\bigcup_{\alpha \in Y} G_{\alpha}$ and define a binary operation * on $S_{Y}$ as: $a_{\alpha} * \dot{b}_{\beta}=\phi_{\alpha, \alpha \beta}\left(a_{\alpha}\right) \phi_{\beta, \alpha \beta}\left(b_{\beta}\right)$ for $a_{\alpha} \in G_{\alpha}, b_{\beta} \in G_{\beta}$.

Then $S_{Y}$ with multiplication * is an inverse semigroup and a union of groups. We denote this semigroup by $\left[Y ; G_{\alpha}(\alpha \in Y) ; \phi_{\alpha, \beta}(\alpha>\beta)\right]$ or simply by $\left[Y ; G_{\alpha} ; \phi_{\alpha, \beta}\right\rfloor$. Any semigroup which is an inverse semigroup and a union of groups can be constructed in this manner ( $\mathrm{cf}$. Theorem 4.11 , 
[3]).

REMARK. Henceforth we will identify an element $\alpha$ of $Y$ with the identity element of $G_{\alpha}$. This means that $Y$ can be identified with the semilattice of idempotents of $\left[y ; G_{\alpha} ; \phi_{\alpha, \beta}\right]$. We will also write $a_{\alpha} b_{\beta}$ instead of $a_{\alpha} * b_{\beta}$.

It is clear from the above construction that for any $\alpha, \beta \in Y$, $G_{\alpha \beta} G_{\beta} \subseteq G_{\alpha \beta}$. The following facts are well known ( $\mathrm{ff}$. Lemmas 4.8 and 4.9 , [3]):

A. if $\alpha \in Y$ then $\alpha$ commutes with every element of $S_{Y}$,

B. if $\alpha>\beta$ and $a_{\alpha} \in G_{\alpha}$ then $\phi_{\alpha, \beta}\left(a_{\alpha}\right)=a_{\alpha}{ }^{\beta}$.

The above items will be used repeatedly without mention in the proof of Theorem 4 .

THEOREM 4 (CIifford [2]). There is an isomorphism

$$
i:\left[Y ; G_{\alpha} ; \phi_{\alpha, \beta}\right] \rightarrow\left[Y^{\prime} ; \hat{G}_{\gamma} ; \hat{\phi}_{Y, \sigma}\right]
$$

if and only if there is an isomorphism $\psi: Y \rightarrow Y^{\prime}$ and isomorphisms $i_{\alpha}: G_{\alpha} \rightarrow \hat{G}_{\psi(\alpha)}(\alpha \in Y)$ such that if $\alpha>\beta(\alpha, \beta \in Y)$ then $\hat{\phi}_{\psi(\alpha), \psi(\beta)_{\alpha}}=i_{\beta} \phi_{\alpha, \beta}$.

Proof of necessity. Let $\psi$ be the restriction of $i$ to $Y$. Let $i_{\alpha}$ be the restriction of $i$ to $G_{\alpha}(\alpha \in Y)$. Then $i$ and $i_{\alpha}$ are isomorphisms onto $Y^{\prime}$ and $\hat{G}_{\psi(\alpha)}$ respectively.

If $\alpha>\beta$ in $Y$ and $\alpha_{\alpha} \in G_{\alpha}$ then

$$
\begin{aligned}
i_{\beta}\left[\phi_{\alpha, \beta}\left(a_{\alpha}\right)\right]=i_{\beta}\left(a_{\alpha} \beta\right)=i\left(a_{\alpha} \beta\right)=i\left(a_{\alpha}\right) & i(\beta)= \\
& =i_{\alpha}\left(a_{\alpha}\right) \psi(\beta)=\hat{\phi}_{\psi(\alpha), \psi(\beta)}\left[i_{\alpha}\left(a_{\alpha}\right)\right] .
\end{aligned}
$$

Proof of sufficiency. For $a_{\alpha} \in G_{\alpha}$ define $i\left(a_{\alpha}\right)=i_{\alpha}\left(a_{\alpha}\right)$. Let $a_{\alpha} \in G_{\alpha}$ and $a_{\beta} \in G_{\beta}$. Then 


$$
\begin{aligned}
i\left(a_{\alpha \beta} b_{\beta}\right) & =i_{\alpha \beta}\left(a_{\alpha \beta} b_{\beta}\right) \\
& =i_{\alpha \beta}\left(a_{\alpha} b_{\beta}\right) \psi(\alpha \beta) \\
& =i_{\alpha \beta}\left(a_{\alpha \beta} b_{\beta}\right) i_{\alpha \beta}(\alpha \beta) \\
& =i_{\alpha \beta}\left(a_{\alpha} b_{\beta}^{\alpha \beta}\right) \\
& =i_{\alpha \beta}\left(a_{\alpha} \alpha \beta b_{\beta}^{\alpha \beta}\right) \\
& =i_{\alpha \beta}\left[\phi_{\alpha, \alpha \beta}\left(a_{\alpha}\right) \phi_{\beta, \alpha \beta}\left(b_{\beta}\right)\right] \\
& =\left[\left(i_{\alpha \beta} \phi_{\alpha, \alpha \beta}\right) a_{\alpha}\right]\left[\left(i_{\alpha \beta} \phi_{\beta, \alpha \beta}\right) b_{\beta}\right] \\
& =\left[\hat{\phi}_{\psi(\alpha), \psi(\alpha \beta)}\left(i_{\alpha}\left(a_{\alpha}\right)\right)\right]\left[\hat{\phi}_{\psi(\beta), \psi(\alpha \beta)}\left(i_{\beta}\left(b_{\beta}\right)\right)\right] \\
& =\left[i_{\alpha}\left(a_{\alpha}\right) \psi(\alpha \beta)\right]\left[i_{\beta}\left(b_{\beta}\right) \psi(\alpha \beta)\right] \\
& =i_{\alpha}\left(a_{\alpha}\right) i_{\beta}\left(b_{\beta}\right) \\
& =i_{\left.\left(a_{\alpha}\right) i_{(b \beta}\right) .}
\end{aligned}
$$

This completes the proof of Theorem 4.

The proof of the next theorem follows easily from the equivalence of parts 1 and 6 of Theorem 3 [6] and from Theorems 3 and 4 above.

THEOREM 5. A semigroup $S$ is commutative and categorical if and only if there exists a categorical semilatice $Y$ and abelian groups $G_{\alpha}$ $(\alpha \in Y)$ such that $S=\left(\left(\left[Y ; G_{\alpha} ; \phi_{\alpha, \beta}\right] ; A_{x}\right)\right)$ for some collection of sets $A_{x}, x \in \underset{\alpha \in Y}{U} G_{\alpha} ;$ furthermore, two commutative categorical semigroups $\left(\left(\left[Y ; G_{\alpha} ; \phi_{\alpha, \beta}\right] ; A_{x}\right)\right)$ and $\left(\left(\left[Y^{\prime} ; \hat{G}_{\gamma} ; \hat{\phi}_{\gamma, \sigma}\right] ; B_{y}\right)\right)$ are isomorphic if and only if there exists an isomorphism $\psi: Y \rightarrow Y^{\prime}$ and isomorphisms $i_{\alpha}: G_{\alpha} \rightarrow \hat{G}_{\psi(\alpha)}(\alpha \in Y)$ such that if $\alpha>\beta(\alpha, \beta \in Y)$ then
A. $\hat{\phi}_{\psi(\alpha), \psi(\beta)_{\alpha}}=i_{\beta} \phi_{\alpha, \beta}$ and
B. for any $x \in G_{\alpha}, \quad\left|A_{x}\right|=\left|B_{i_{\alpha}(x)}\right|$.

REMARK. The structure of commutative semigroups which have a regular subsemigroup of products can be obtained modulo the structure of semilattices and abelian groups in a similar manner. Theorems 3 and 4 again give a complete set of invariants. 


\section{An open question}

It follows from Proposition 2 that the partial groupoid $E_{S}$ of idempotents of a regular categorical semigroup $S$ satisfies the following property: if $e \leq g, f \leq g$ for some $e, f, g \in E_{S}$ then there exists $t \in E_{S}$ such that either $t \leq f$ and $t E_{S} t \cong e E_{S} e$ or $t \leq e$ and $t E_{S} t \cong f E_{S} f$

It is an open question as to whether or not this condition is sufficient for a partial band to appear as an isomorphic copy of the partial groupoid of idempotents of some regular categorical semigroup.

\section{References}

[1] G.R. Baird, "On semigroups and uniform partial bands", Semigroup Forum 4 (1972), 185-188.

[2] A.H. Clifford, "Semigroups admitting relative inverses", Ann. of Math. 42 (1941), 1037-1049.

[3] A.H. Clifford and G.B. Preston, The algebraic theory of semigroups, Vol. I (Math. Surveys 7 (I), Amer. Math. Soc., Providence, Rhode Island, 1961).

[4] T.E. Hall, "On orthodox semigroups and uni form and antiuniform bands", J. Algebra 16 (1970), 204-217.

[5] F.R. McMorris and M. Satyanarayana, "Categorical semigroups", Proc. Amer. Math. Soc. 33 (1972), 271-277.

[6] R.A.R. Monzo, "Categorical semigroups", Semigroup Forwo 6 (1973), 59-68.

[7] Mario Petrich, "On a class of completely semisimple inverse semigroups", Proc. Amer. Math. Soc. 24 (1970), 671-676. 
[8] Mario Petrich, "Regular semigroups satisfying certain conditions on idempotents and ideals", Trans. Amer. Math. Soc. 170 (1972), $245-268$.

Department of Mathematics, Institute of Advanced Studies, Australian National University, Canberra, ACT. 